\title{
Genome-wide (over)view on the actions of vitamin D
}

\section{Carsten Carlberg*}

School of Medicine, Institute of Biomedicine, University of Eastern Finland, Kuopio, Finland

\section{Edited by:}

Debra Diz, Wake Forest Universtiy

School of Medicine, USA

\section{Reviewed by:}

Moray J. Campbell, Roswell Park

Cancer Institute, USA

Gregory A. Hawkins, Wake Forest

University Health Sciences, USA

*Correspondence:

Carsten Carlberg, School of Medicine, Institute of Biomedicine, University of Eastern Finland, Room 3179, Yliopistonranta 1 E, PO Box 1627, Fl-70211 Kuopio, Finland e-mail: carsten.carlberg@uef.fi
For a global understanding of the physiological impact of the nuclear hormone $1 \alpha, 25$-dihydroxyvitamin $\mathrm{D}_{3}\left(1,25(\mathrm{OH})_{2} \mathrm{D}_{3}\right)$ the analysis of the genome-wide locations of its high affinity receptor, the transcription factor vitamin D receptor (VDR), is essential. Chromatin immunoprecipitation sequencing (ChIP-seq) in GM10855 and GM10861 lymphoblastoid cells, undifferentiated and lipopolysaccharide-differentiated THP-1 monocytes, LS180 colorectal cancer cells and LX2 hepatic stellate cells revealed between 1000 and 13,000 VDR-specific genomic binding sites. The harmonized analysis of these ChIP-seq datasets indicates that the mechanistic basis for the action of the VDR is independent of the cell type. Formaldehyde-assisted isolation of regulatory elements sequencing (FAIRE-seq) data highlight accessible chromatin regions, which are under control of $1,25(\mathrm{OH})_{2} \mathrm{D}_{3}$. In addition, public data, such as from the ENCODE project, allow to relate the genome-wide actions of VDR and $1,25(\mathrm{OH})_{2} \mathrm{D}_{3}$ to those of other proteins within the nucleus. For example, locations of the insulator protein CTCF suggest a segregation of the human genome into chromatin domains, of which more than 1000 contain at least one VDR binding site. The integration of all these genome-wide data facilitates the identification of the most important VDR binding sites and associated primary $1,25(\mathrm{OH})_{2} \mathrm{D}_{3}$ target genes. Expression changes of these key genes can serve as biomarkers for the actions of vitamin $D_{3}$ and its metabolites in different tissues and cell types of human individuals. Analysis of primary tissues obtained from vitamin $D_{3}$ intervention studies using such markers indicated a large inter-individual variation for the efficiency of vitamin $D_{3}$ supplementation. In conclusion, a genome-wide (over)view on the genomic locations of VDR provides a broader basis for addressing vitamin D's role in health and disease.

Keywords: vitamin D, vitamin D receptor, chromatin, gene regulation, epigenomics, genomics

\section{INTRODUCTION}

During evolution the secosteroid vitamin $\mathrm{D}_{3}$ became a pleiotropic signaling molecule (Jones et al., 1998). Initially, the molecule was used by early unicellular organisms to protect their DNA against UV-B irradiation (Holick, 2011). Far later, when the first fish with bones evolved, the endocrinology of vitamin $\mathrm{D}_{3}$ was established, and still is very conserved in all higher organisms, including humans (Bouillon and Suda, 2014). In this system, the energy of UV-B is used to convert 7-dehydrocholesterol into

\footnotetext{
Abbreviations: $1,25(\mathrm{OH})_{2} \mathrm{D}_{3}, \quad 1 \alpha, 25$-dihydroxyvitamin $\mathrm{D}_{3} ; \quad 25(\mathrm{OH}) \mathrm{D}_{3}, \quad 25$ hydroxyvitamin $\mathrm{D}_{3}$; ALOX5, arachidonate 5-lipoxygenase; CBS, cystathionine $\beta$-synthase; CCNC, cyclin C; CDKN1A, cyclin-dependent kinase inhibitor 1A; CHD7, chromodomain helicase DNA binding protein 7; ChIA-PET, chromatin interaction analysis by paired-end tag sequencing; ChIP, chromatin immunoprecipitation; ChIP-seq, ChIP coupled with massive parallel sequencing; CTCF, CCCTC-binding factor; CYP, cytochrome P450; DNase-seq, DNase I hypersensitivity sites sequencing; DR3, direct repeat spaced by 3 nucleotides; FAIRE-seq, formaldehyde-assisted isolation of regulatory elements sequencing; IGV, Integrative Genomics Viewer; LPS, lipopolysaccharide; LRP5, low density lipoprotein receptor-related protein 5; MYC, v-myc avian myelocytomatosis viral oncogene homolog; PBMC, peripheral blood mononuclear cell; RXR, retinoid $\mathrm{X}$ receptor; THBD, thrombomodulin; TNFSF11, tumor necrosis factor (ligand) superfamily, member 11; TRPV6, transient receptor potential cation channel, subfamily V, member 6; TSS, transcription start site; VDR, vitamin D receptor; ZMIZ1, zinc finger, MIZ-type containing 1.
}

pre-vitamin $\mathrm{D}_{3}$, i.e., UV-B became essential for the synthesis of vitamin $\mathrm{D}_{3}$ (Holick, 2004) (more details in the article by Reichrath et al. in this issue). The central importance of this step is emphasized by the step-wise depigmentation of human skin, when modern humans started to move out of Africa some 100,000 years ago (Hochberg and Templeton, 2010). Two hydroxylation steps are necessary for the conversion of vitamin $\mathrm{D}_{3}$ via 25-hydroxyvitamin $\mathrm{D}_{3}\left(25(\mathrm{OH}) \mathrm{D}_{3}\right)$ into the biologically active vitamin $\mathrm{D}_{3}$ metabolite, $1,25(\mathrm{OH})_{2} \mathrm{D}_{3}$ (Norman, 2008). The latter molecule participates in a large number of physiological processes, such as bone formation, immune function and cellular growth and differentiation (Deluca, 2004) (more details in the articles by van Leeuwen et al., Hewison et al. and Munoz et al. in this issue).

The transcription factor VDR is the only high-affinity target for $1,25(\mathrm{OH})_{2} \mathrm{D}_{3}$ within the cell nucleus (Haussler et al., 1997). VDR is one of approximately 1900 transcription factors, which are encoded by the human genome (Vaquerizas et al., 2009). In addition, VDR is a member of the superfamily of nuclear receptors, most of which are specifically activated by lipophilic molecules in the size of cholesterol (Carlberg and Molnár, 2012). Its lipophilic allows $1,25(\mathrm{OH})_{2} \mathrm{D}_{3}$ to pass through all biological membranes, i.e., gene regulation by vitamin $\mathrm{D}$ 
does not involve additional signal transduction steps, as they are known for hydrophilic signaling molecules, such as peptide hormones, growth factors and cytokines. Moreover, VDR is rather ubiquitously expressed, i.e., most human tissues and cell types are responsive to $1,25(\mathrm{OH})_{2} \mathrm{D}_{3}$ (Wang et al., 2012).

VDR shares the main structural characteristics of nuclear receptors, which is a highly conserved DNA-binding domain and a structurally conserved ligand-binding domain (Mangelsdorf et al., 1995). VDR's DNA-binding domain specifically contacts the hexameric consensus sequence RGKTSA ( $R=A$ or $G, K=G$ or $\mathrm{T}, \mathrm{S}=\mathrm{C}$ or $\mathrm{G}$ ) within the major groove of genomic DNA (Shaffer and Gewirth, 2002). However, like most other transcription factors, VDR uses a partner DNA-binding protein, in order to bind efficiently to its target sites. More than 20 years ago, this heterodimeric partner turned out to be the nuclear receptor retinoid X receptor (RXR) (Sone et al., 1991; Carlberg et al., 1993). Steric constraints of the dimerizing DNA-binding domains of VDR and RXR determine the optimal binding site of the VDRRXR complex as a direct repeat of two hexameric nuclear receptor binding motifs spaced by three nucleotides (DR3) (Umesono et al., 1991; Shaffer and Gewirth, 2004). Within VDR's ligandbinding domain, a network of some 40 mostly non-polar amino acids forms a ligand-binding pocket, in which $1,25(\mathrm{OH})_{2} \mathrm{D}_{3}$ and its synthetic analogs are specifically fixed with high affinity (Molnár et al., 2006). This ligand binding process induces a conformational change to the surface of VDR's ligand-binding domain, which results in a significant change of VDR's proteinprotein interaction profile: it transforms from a repressor to an activator (Moras and Gronemeyer, 1998; Carlberg and Campbell, 2013) (more details on VDR structure in the article by Molnar in this issue).

Taken together, vitamin D signaling primarily comprises the molecular actions of the VDR, i.e., the physiological effects of $1,25(\mathrm{OH})_{2} \mathrm{D}_{3}$ are largely identical to those of its receptor. This reduces vitamin $\mathrm{D}$ signaling to one central question: which are the most important genomic targets of VDR in a given tissue and which genes are controlled via these sites? Thus, this review focuses on the description of the genome-wide binding of VDR and its mechanistic implications. This analysis will be in the context of genome-wide information on chromatin accessibility and the presence of other nuclear proteins, such as provided by the ENCODE consortium.

\section{GENOME-WIDE VDR BINDING}

The method chromatin immunoprecipitation (ChIP) was developed, in order to monitor the binding of transcription factors to their genomic targets (Orlando, 2000). The core of the method is (i) mild chemical cross-linking of living cells or tissues, e.g., with $1 \%$ formaldehyde, in order to fix nuclear proteins to genomic DNA, (ii) sonication of chromatin into small (200-400 bp) fragments, and (iii) immunoprecipitation with an antibody specific for the chosen nuclear protein (Maston et al., 2012). In this way, chromatin regions, which, at the moment of cross-linking, had been in contact with the protein of choice, are specifically enriched. A specific ChIP signal, in reference to a control (often unspecific $\operatorname{IgGs}$ ), is a strong indication that the protein of choice had been in contact with the selected genomic region at the moment of cross-linking.

At earlier times, the isolated chromatin template was analyzed by site-specific quantitative PCR (ChIP-qPCR). This approach had been used to study, for example, the extended promoter regions of the primary VDR target genes CYP24A1 (Väisänen et al., 2005), CYP27B1 (Turunen et al., 2007), CCNC (Sinkkonen et al., 2005), and CDKN1A (Saramäki et al., 2006, 2009). Alternatively, the abundance of immunoprecipitated chromatin fragments had been detected by tiled microarrays (so-called "chips,") which covered a selection of promoter and enhancer regions or any other subset of the genome (ChIP-chip). The group of Pike et al. had extensively used ChIP-chip, in order to locate VDR binding sites within the regulatory regions of the mouse genes $V d r$ (Zella et al., 2006), Trpv6 (Meyer et al., 2006), Lrp5 (Fretz et al., 2007), Tnfsf11 (also known as Rankl) (Kim et al., 2006), Cyp24a1 (Meyer et al., 2010), and Cbs (Kriebitzsch et al., 2011). The latest development of the ChIP method is the unbiased analysis of the precipitated chromatin by massively parallel DNA sequencing (ChIP-seq), i.e., the detection of the binding sites of the transcription factor of choice in the complete genome. To date, ChIP-qPCR is primarily used for the confirmation of ChIP-seq results, while ChIP-chip got outdated shortly after its introduction. This leaves, at present, ChIP-seq as the method of choice for analyzing VDR's genomic binding loci.

At present, the readouts of massive parallel sequencing are small sequence tags (35-50 nucleotides), but in the future there will be in majority longer reads used, which will lead to improved significance of the results. These sequence tags are aligned to a reference genome (for human samples this is, at present, hg19) and specifically represent the enriched chromatin fragments. Then "peak calling" software is used to identify genomic regions, in which significantly more sequence tags are detected than in control reactions. Therefore, tags that accumulate as "peaks" at specific genomic loci mark the presence of the investigated nuclear protein (Park, 2009; Furey, 2012). At present, ChIP is still performed with millions of cells; in case of a prominent binding site, most of these cells contribute to the ChIP signal, i.e., it can be assumed that in the majority of cells the locus is occupied by VDR. However, when only in some cells a site is bound by VDR, the respective peak is far less prominent, i.e., most likely of less impact for the regulation of $1,25(\mathrm{OH})_{2} \mathrm{D}_{3}$ target genes.

To date, VDR ChIP-seq data are available from (i) the immortalized lymphoblastoid cell lines GM10855 and GM10861 (Ramagopalan et al., 2010), (ii) undifferentiated THP-1 monocyte-like cells (Heikkinen et al., 2011), (iii) lipopolysaccharide (LPS)-polarized THP-1 macrophage-like cells (Tuoresmäki et al., 2014), (iv) LS180 colorectal cancer cells (Meyer et al., 2012), and (v) LX2 hepatic stellate cells (Ding et al., 2013). The original publications reported between 1600 and 6200 VDR binding sites (in ligand-stimulated samples) within the human genome. However, these numbers are not directly comparable, since different peak calling software, alternative threshold settings and even an older version of the reference genome (hg18) were used. A harmonized re-analysis of all six VDR ChIP-seq datasets with identical peak calling settings (MACS, version 2) resulted for $1,25(\mathrm{OH})_{2} \mathrm{D}_{3}$-stimulated and unstimulated cells, respectively, 
in following number of binding sites: 6172 and 3144 (GM10855), 12,353 and 4072 (GM10861), 774 and 609 (undifferentiated THP-1), 953 and 529 (LPS-differentiated THP-1), 3777 and 165 (LS180) and 1532 and 1474 (LX2) (Tuoresmäki et al., 2014).

In total, the six VDR ChIP-seq datasets indicated 21,776 nonoverlapping VDR binding sites when allowing a distance of up to 250 bp between the peak summits (Tuoresmäki et al., 2014). However, the vast majority of these VDR loci (67\%) are unique for one of the analyzed cellular models. In contrast, under the above mentioned conditions only 54 sites are common within all six datasets. In general, this indicates that VDR displays a very individual pattern of cell-specific genomic locations, which overlaps between multiple tissues only at key sites. The VDR binding site of the $1,25(\mathrm{OH})_{2} \mathrm{D}_{3}$ target gene $Z M I Z 1$, which is located $15.3 \mathrm{~kb}$ downstream of the transcription start site (TSS), represents an example of such a locus (Figure 1). In general, the rates of overlaps between the cell types follow roughly their developmental and functional relatedness, i.e., the two lymphoblastoid cell lines, GM10855 and GM10861, or LPS-differentiated and undifferentiated THP-1 cells show more overlapping VDR binding sites than all other comparisons between the VDR ChIP-seq datasets. Moreover, the VDR binding profiles of ligand-stimulated cells matched better than those of unstimulated cells (Tuoresmäki et al., 2014).

Genome-wide studies on VDR binding have changed the view on vitamin $\mathrm{D}$ signaling. The few dozens rather well characterized VDR binding sites in less than $10 \mathrm{~kb}$ distance to the TSS of $1,25(\mathrm{OH})_{2} \mathrm{D}_{3}$ target genes (Haussler et al., 2013), which were known before, were complemented by thousands of additional VDR loci spread over the whole genome. However, the very most of the loci, which were highlighted by ChIP-seq, have not yet been validated by ChIP-qPCR or similar methods (and many will never be confirmed). Some previously known VDR binding sites, such as those controlling the genes MYC (Toropainen et al., 2010), VDR (Zella et al., 2010), CCNC (Sinkkonen et al., 2005), and ALOX5 (Seuter et al., 2007), could be confirmed by the VDR ChIP-seq datasets. However, for many known $1,25(\mathrm{OH})_{2} \mathrm{D}_{3}$ target genes the ChIP-seq data suggest additional or alternative VDR binding sites, many of these being far more distant to the gene's TSS region than previously foreseen. In the past, many of these VDR binding sites had been overlooked due to a focus to only a few kb upstream of the TSS of $1,25(\mathrm{OH})_{2} \mathrm{D}_{3}$ target genes. However, in accordance with the results of the ENCODE project (ENCODE-Project-Consortium et al., 2012), VDR binding sites are found with equal probability upstream and downstream of the TSS region of $1,25(\mathrm{OH})_{2} \mathrm{D}_{3}$ target genes. In addition, VDR loci in distance of even more than $1 \mathrm{Mb}$ from the gene's TSS are accepted as regulatory sites (more details below).

In summary, there seem to be 1000-10,000 genomic VDR binding sites per cell type. This is far more than the number of primary $1,25(\mathrm{OH})_{2} \mathrm{D}_{3}$ target genes, which is in the order of $100-500$ per tissue. This even holds true for undifferentiated THP-1 cells, where 774 VDR loci in ligand-stimulated cells are facing 408 statistically significantly up-regulated early $1,25(\mathrm{OH})_{2} \mathrm{D}_{3}$ responding genes (Heikkinen et al., 2011). The indicates that some genes are controlled by more than one VDR binding site, i.e., they may have a higher potential to be regulated by $1,25(\mathrm{OH})_{2} \mathrm{D}_{3}$ than target genes with only one active VDR locus (more details on the transcriptome-wide response to $1,25(\mathrm{OH})_{2} \mathrm{D}_{3}$ in the article by Campbell et al. in this issue).

\section{MECHANISTIC INSIGHT FROM VDR ChIP-SEO STUDIES}

The close to 22,000 non-overlapping VDR peaks, which are indicated by the public ChIP-seq datasets (Tuoresmäki et al., 2014), show rather different characteristics. Despite the rather different total number of reported VDR peaks per cellular model, each of the six ChIP-seq datasets contains an in part overlapping subset of less than 200 sites, where a stimulation with $1,25(\mathrm{OH})_{2} \mathrm{D}_{3}$ resulted in a significant increase of VDR binding compared to unstimulated samples. These VDR loci are far more prominent than most of the other sites, for which ligand treatment was either repressive, had no effect or was only minor stimulatory.

Another important parameter for the characterization of a VDR binding site is the presence or absence of a high confidence DR3-type binding site below the summit ( $\pm 100 \mathrm{bp})$ of the respective ChIP-seq peak. This can be investigated with the help of binding site screening algorithms, such as provided by HOMER (Heinz et al., 2010). Depending on the threshold settings the software detects binding sites that deviate more or less from the consensus sequence. For example, for a moderate setting of a HOMER score of 7, from the total of 21,776 non-overlapping VDR sites in all six ChIP-seq datasets only 3801 (17.5\%) contain a DR3-type sequence. Interestingly, the percentage of DR3-type motifs differs significantly between the datasets and ranges from $38.2 \%$ (483 of 1264 sites) in LPS-polarized THP-1 cells via 36.4\% (373 of 1023) in undifferentiated THP-1 cells, $28.6 \%$ (1062 of 3706 ) in LS180 cells, $27.8 \%$ (611 of 2194) in LX2 cells, $13.0 \%$ (909 of 6975 ) in GM10855 cells to $9.0 \%$ (1118 of 12,438) in GM10861 cells. This indicates that the total number of identified VDR binding sites in each cell line inversely correlates with the percentage of peak summits with DR3-type sites. However, when the analysis is restricted to the top 200 VDR sites (based on fold enrichment scoring), for all six ChIP-seq datasets a DR3-like sequence rate of more than $60 \%$ is observed, i.e., DR3 motifs are found preferentially at highly ligand responsive VDR loci. In this way, the different VDR ChIP-seq datasets show a very similar relationship between VDR occupancy and DR3 percentage. This suggests that the mechanistic basis for the action of the VDR is independent of the cell type and the total number of identified binding sites.

Transcription factor binding site screening software, such as HOMER, suggests that DR3-type binding sequences are the most abundant sites below the summits of VDR ChIP-seq peaks. However, a significant number of the genomic VDR loci (depending on the dataset $60-90 \%$ of all, see above) do not associate with a DR3-type site. This indicates that at these loci VDR uses a different mode of interaction with genomic DNA. This could be either the use of a different heterodimeric binding partner or an indirect binding "backpack" of a DNA-binding transcription factor (Carlberg and Campbell, 2013). In both scenarios the specific DNA binding site would be different to a DR3-type sequence. Interestingly, for the VDR ChIP-seq datasets originating from hematopoietic cells, HOMER indicated binding sites for the transcription factors PU.1 (also called SPI1), ESRRB (also called NR3B2) and GABPA as significantly enriched (Tuoresmäki 


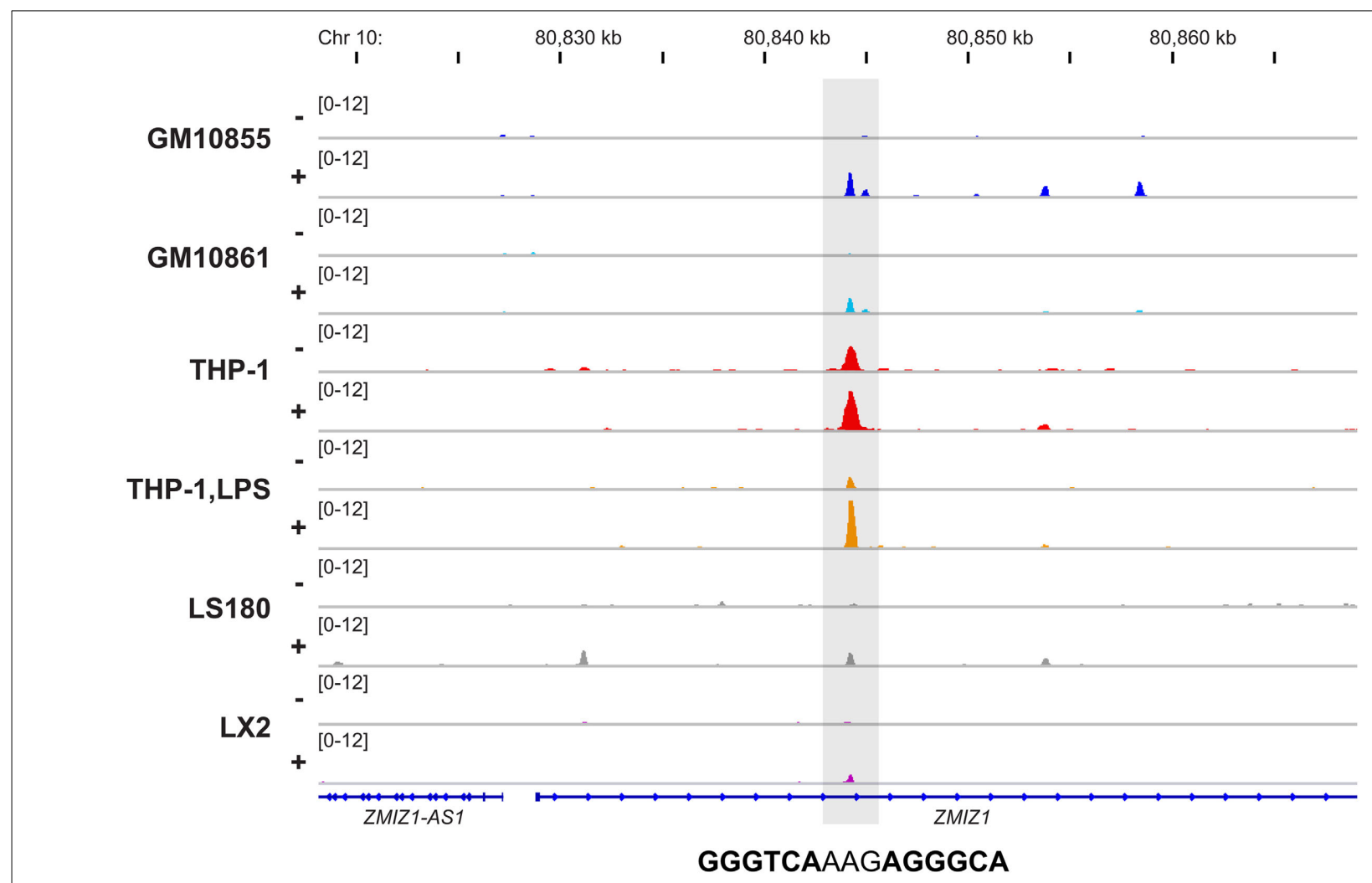

FIGURE 1|Conserved genomic VDR binding in six cellular models. The Integrative Genomics Viewer (IGV) browser (Robinson et al., 2011) was used to visualize the VDR binding site $15.3 \mathrm{~kb}$ downstream of the ZMIZ1 TSS. The peak tracks display data from VDR ChIP-seq datasets from two B cell-like cells (dark and light blue), monocyte-like cells (red), macrophage-like cells (orange), colon cells (gray) and liver cells (violet). The cells were either unstimulated $(-)$ or treated with VDR ligand $(+)$. The gene structures are shown in blue and the sequence of the DR3-type element below the summit of the VDR peak is indicated. et al., 2014). PU.1 is well-known as a pioneer factor (Zaret and Carroll, 2011), i.e., as a transcription factor with (i) a high number of genomic binding sites, (ii) a greater binding promiscuity and (iii) higher diversity of interactions. Pioneer factors are the first that bind regulatory genomic regions, such as promoters and enhancers, and interact with chromatin modifying enzymes, in order make the chromatin more accessible for regular transcription factors, such as VDR. At present, a direct protein-protein interaction of VDR with PU.1, ESRRB or GABPA has not been demonstrated, but for the Ets family, to which PU.1 belongs, there were indications for an interaction (Tolon et al., 2000). However, for the pure function as a pioneer factor a direct protein-protein interaction with the "settler factor" is not needed. Moreover, there is older evidence from single gene studies that DNA binding of VDR is modulated by the transcription factors AP1 (Schüle et al., 1990) and RUNX2 (Sierra et al., 2003). In contrast, a genomewide study on the interaction of VDR with the transcription factor TCF7L2 did not provide any evidence that the latter acts as a pioneer factor for VDR (Meyer et al., 2012).

Below VDR peak summits no dominant non-DR3 binding sequence could be identified. Moreover, the six VDR ChIP-seq datasets differ in the ranking and identity of the non-DR3 sites found below the peaks (Tuoresmäki et al., 2014). This suggests that in total there must be a larger number of VDR partnering proteins. Most likely, these proteins have a cell-specific expression pattern and may explain in part the cell-specific actions of VDR and its natural ligand $1,25(\mathrm{OH})_{2} \mathrm{D}_{3}$. Moreover, ChIP-seq datasets have indicated that, in contrast to steroid receptors, VDR binds a number of its genomic targets already in the absence of ligand. These ligand-independent genomic VDR loci have a clearly lower rate of DR3-type sequences than ligand-dependent sites (Heikkinen et al., 2011). In contrast, they associate preferentially with proteins related to gene repression, such as demonstrated for the example of the CYP27B1 gene (Turunen et al., 2007). This implies that the functional profile of VDR is larger than that of its ligand (Polly et al., 2000) as previously shown for other members of the nuclear receptor superfamily, such as thyroid hormone receptor or liver X receptor (Perissi et al., 2010).

Taken together, all VDR ChIP-seq studies confirm the preferential binding of VDR to DR3-type sequences. However, only one in six of some 22,000 presently known VDR loci within the human genome carry a DR3 site. Thus, there have to be additional mechanisms for the association of VDR with its genomic loci, which may include partnering with presently undefined partner proteins or the tethering to other DNA-binding transcription factors, such as pioneer factors. These should explain some of the cell-specific actions as well as repressive functions of $1,25(\mathrm{OH})_{2} \mathrm{D}_{3}$ and the VDR. 


\section{RESPONSES OF CHROMATIN TO $1,25(\mathrm{OH})_{2} \mathrm{D}_{3}$}

Histone proteins forming the nucleosome core are DNAbinding proteins but do not show any sequence specificity. Therefore, the complex of nucleosomes and genomic DNA, which is referred to as chromatin, has an intrinsic repressive potential: it prevents access of transcription factors to their genomic targets (Razin, 1998). This provides essential stability to the epigenetic landscape for long-lasting regulatory decisions, such as gene expression in terminally differentiated cells (Mohn and Schubeler, 2009). In contrast, some regions of the epigenome show highly dynamic changes in response to extra- and intracellular signals, such as the activation of VDR by $1,25(\mathrm{OH})_{2} \mathrm{D}_{3}$ binding (Talbert and Henikoff, 2006). These changes involve the methylation of genomic DNA and/or reversible post-translational modifications of histone proteins, such as acetylation or deacetylation at exposed lysine residues (Narlikar et al., 2002). Dynamic chromatin modifications change the access to regulatory genomic regions, such as promoter and enhancers, for the binding of transcription factors, i.e., they determine whether at these regions chromatin is open or closed. This can be monitored genome-wide by using the method DNase I hypersensitive sites sequencing (DNase-seq), which highlights genomic regions being most sensitive to cleavage by the enzyme DNase I (Crawford et al., 2006). A very similar technique is Formaldehyde-Assisted Isolation of Regulatory Elements sequencing (FAIRE-seq), which identifies genome-wide accessible DNA regions (Giresi et al., 2007) (more details on the relation of the epigenome and $1,25(\mathrm{OH})_{2} \mathrm{D}_{3}$ in the article by Kallay et al. in this issue).

At present, the only publically available dataset describing genome-wide effects of $1,25(\mathrm{OH})_{2} \mathrm{D}_{3}$ on the epigenome, is a detailed FAIRE-seq time course in THP-1 cells (Seuter et al., 2013). These data demonstrate that some $87 \%$ of the more than 1000 VDR binding sites in this cellular model co-localize with open chromatin. Interestingly, at 165 of these VDR loci a strong $1,25(\mathrm{OH})_{2} \mathrm{D}_{3}$-dependent increase of chromatin accessibility is found. Importantly, at $66 \%$ of these chromatin regions a DR3type sequence is found, i.e., they overlap with loci, at which VDR binding is enhanced most by $1,25(\mathrm{OH})_{2} \mathrm{D}_{3}$ stimulation (Seuter et al., 2013). Moreover, the binding of VDR to its genomic loci is a dynamic process, which takes at least some $2 \mathrm{~h}$ to saturate the sites. One example is a site located $225 \mathrm{~kb}$ downstream of the TSS of the chromodomain helicase DNA binding protein 7 (CHD7) gene (Figure 2). It demonstrates that at the same locus, where a strong ligand-dependent increase of VDR binding is observed, the rate of open chromatin more than doubled already $40 \mathrm{~min}$ after incubation of THP-1 cells with $1,25(\mathrm{OH})_{2} \mathrm{D}_{3}$. At some 200 additional VDR binding loci the chromatin shows detectable but less prominent response to $1,25(\mathrm{OH})_{2} \mathrm{D}_{3}$ treatment, while at the remaining 500 sites the VDR ligand did not affect chromatin accessibility. Accordingly, only at less than $20 \%$ of the latter sites DR3-type sequences are found. At many of these sites, VDR binds already in the absence of ligand and may have a different mode of DNA recognition and action (see above).

In summary, at approximately a third of its genome-wide binding loci VDR dynamically controls the epigenetic state of chromatin. At these sites, VDR binding and chromatin opening are tightly interconnected and provide indications for primary $1,25(\mathrm{OH})_{2} \mathrm{D}_{3}$ target genes. This allows a better understanding of the $1,25(\mathrm{OH})_{2} \mathrm{D}_{3}$ signaling cascade.

\section{THE USE OF ENCODE DATA FOR UNDERSTANDING $1,25(\mathrm{OH})_{2} \mathrm{D}_{3}$ SIGNALING}

In addition to the above described $1,25(\mathrm{OH})_{2} \mathrm{D}_{3}$-triggered chromatin open, in the future there will be much more data available on the interaction of VDR and $1,25(\mathrm{OH})_{2} \mathrm{D}_{3}$ with the epigenome. This will include FAIRE-seq and DNase-seq studies in further $1,25(\mathrm{OH})_{2} \mathrm{D}_{3}$-responsive tissues as well as investigations on changes of histone modifications and/or co-factor binding in $1,25(\mathrm{OH})_{2} \mathrm{D}_{3}$-responsive genomic regions. However, already at present existing genome-wide data on the annotation of the genomes of human, mouse and other species can be used. The best example is the large range of data collected by the ENCODE consortium (ENCODE-Project-Consortium et al., 2012). The core of the ENCODE datasets are publically available ChIP-seq results on approximately 100 transcription factors and 20 histone modifications from more than 100 human cellular systems. From the latter, the human monocytic leukemia cell line K562 is represented with highest number of datasets, while the majority of the other cells has not been studied with the same intensity. At present, ENCODE data describe primarily the basal status of cells, i.e., only in a very few cases a stimulation with hormones, growth factor, cytokines or similar molecules had been performed. Neither data on $1,25(\mathrm{OH})_{2} \mathrm{D}_{3}$ stimulations nor VDR ChIP-seq data are comprised in the ENCODE dataset. Nevertheless, the examples shown below will illustrate, how already on this stage ENCODE data are useful for a more detailed understanding of $1,25(\mathrm{OH})_{2} \mathrm{D}_{3}$ signaling.

All six VDR ChIP-seq datasets agree with observations of the ENCODE project that (i) transcription factors bind equally likely both up- and downstream of their target gene TSSs and (ii) the likelihood of detecting functional transcription factor binding sites for a given gene decreases by distance from its TSS region (ENCODE-Project-Consortium et al., 2012). This means that, in relation to the TSS of primary $1,25(\mathrm{OH})_{2} \mathrm{D}_{3}$ target genes, the distribution of the VDR binding sites has a Gaussian shape. In turn, this suggest that on the same chromosome there would be no threshold distance for the interaction between a VDR binding locus and the TSS of a primary $1,25(\mathrm{OH})_{2} \mathrm{D}_{3}$ target gene. However, there are limitations provided by higher-order structures of chromatin.

Chromatin forms loops (Kadauke and Blobel, 2009), which contribute to many nuclear functions, such as the control of gene expression (Misteli, 2007). Chromatin loops segregate each chromosome into domains, which are separated by an insulator region (Van Bortle and Corces, 2013). Most insulator regions are associated with the highly conserved transcription factor CCCTCbinding factor (CTCF) (Schmidt et al., 2012). Therefore, ChIPseq data for CTCF binding from multiple human cell lines, such as provided by ENCODE (ENCODE-Project-Consortium et al., 2012), allow a first estimation of the chromatin domain borders (Figure 3). However, only 15-20\% of all genomic CTCF binding sites are involved in insulator function. The method chromatin interaction analysis by paired-end tag sequencing (ChIA-PET) 


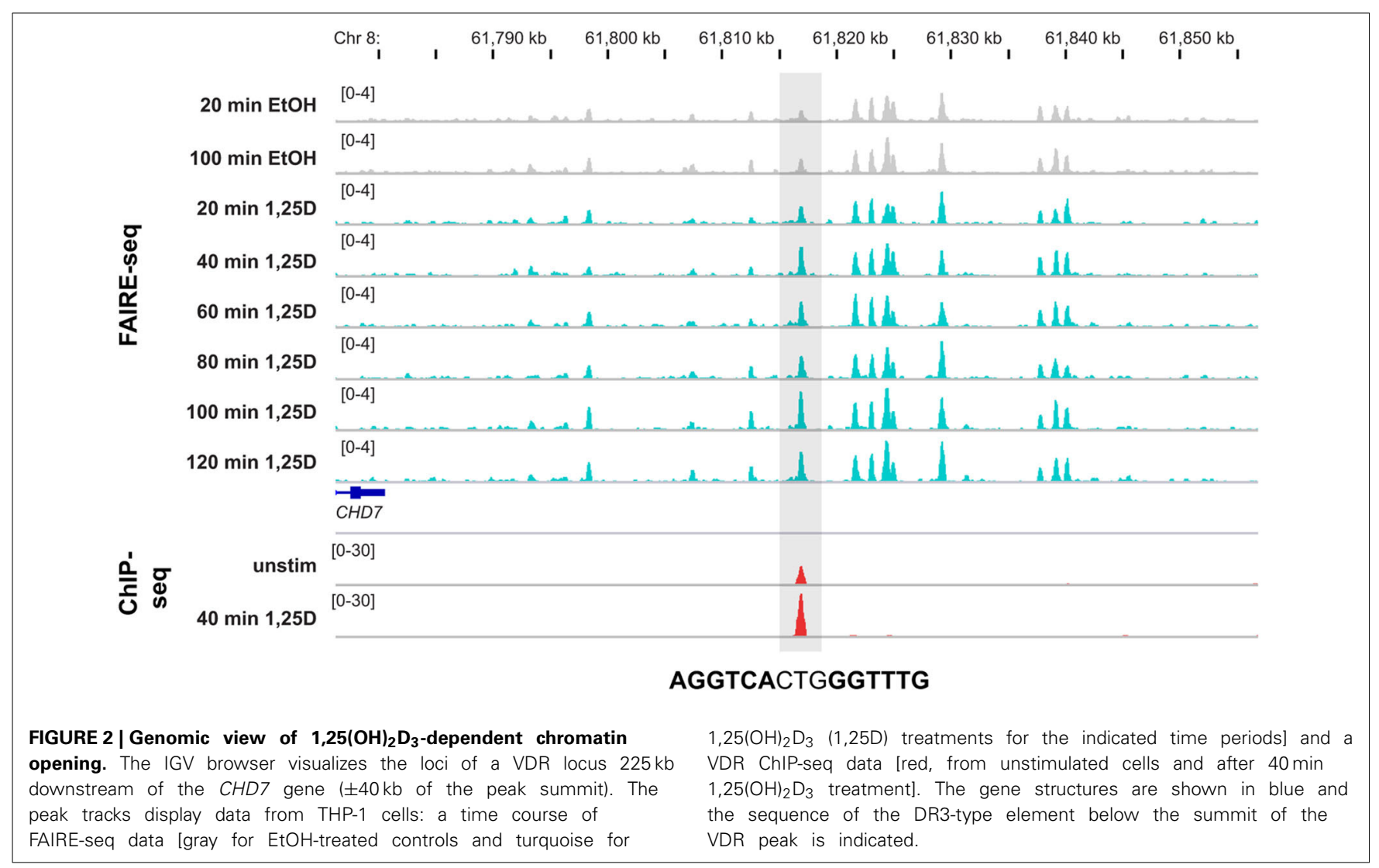

(Fullwood et al., 2009) allows an assessment of the 3-dimensional structure of chromatin. When applied for CTCF in K562 cells it mapped more than 120,000 intra-chromosomal, CTCF-mediated chromatin interactions (ENCODE-Project-Consortium et al., 2012). The high conservation of CTCF binding sites allows a reliable extrapolation of the CTCF ChIA-PET data from K562 cells to THP-1 cells, for which VDR ChIP-seq data is available. The combination of both datasets suggests that in THP-1 cells there are some 1600 chromatin domains, which contain at least one VDR binding site (Seuter et al., 2014). When the TSS region of a gene is within one of these chromatin regions, it may be a primary $1,25(\mathrm{OH})_{2} \mathrm{D}_{3}$ target. In case of the CD14 gene, CTCF ChIA-PET data from K562 cells defined a chromatin domain spanning from $1.5 \mathrm{~kb}$ upstream to $57 \mathrm{~kb}$ downstream of the gene's TSS (Figure 3). This domain spans over the whole CD14 gene and comprises two VDR binding sites 24 and $26 \mathrm{~kb}$ downstream of the gene's TSS. This provides a straightforward gene regulatory scenario explaining (i) the primary response of CD14 to $1,25(\mathrm{OH})_{2} \mathrm{D}_{3}$ and (ii) why the neighboring genes of $C D 14$ do not respond to VDR ligand treatment.

The chromatin domain of the CD14 gene is with a size of less than $60 \mathrm{~kb}$ rather small (Figure 3). In contrast, one of the largest VDR-containing chromatin domains spans over $2.3 \mathrm{Mb}$ of chromosome 8 and combines the MYC gene with four VDR binding sites, of which the most prominent is $1215 \mathrm{~kb}$ of the gene's TSS (Ryynänen et al., 2013). This suggests that under the condition of sufficiently large chromatin domains, gene regulation by VDR can be over a distance of more than $1 \mathrm{Mb}$.
At present, there is no VDR ChIA-PET data available but will come in the future. However, via the ENCODE experiment matrix (http://encodeproject.org/ENCODE/dataMatrix/ encodeDataMatrixHuman.html) there is access to ChIA-PET data for RNA polymerase II in HeLa human cervix carcinoma and MCF-7 human breast carcinoma and for estrogen receptor $\alpha$ in MCF-7 cells. The latter may be of special interests for the breast cancer field.

Taken together, public ENCODE data are important tools, which can be used in combination with genome-wide data on VDR for an extrapolation on the 3-dimensional organization of gene regulation by $1,25(\mathrm{OH})_{2} \mathrm{D}_{3}$.

\section{$1,25(0 \mathrm{OH})_{2} \mathrm{D}_{3}$ TARGET GENES AS BIOMARKERS FOR THE VITAMIN D STATUS OF HUMAN INDIVIDUALS}

In contrast to a number of other nuclear receptor ligands, such as cortisol or estrogen, the endocrinology of $1,25(\mathrm{OH})_{2} \mathrm{D}_{3}$ does not imply any fast changes (Deluca, 2004; Norman, 2008). Under normal circumstances, either the production in UV-B exposed skin or the intake of from diet or supplements should provide sufficient amounts of vitamin $\mathrm{D}_{3}$, in order to achieve optimal serum $25(\mathrm{OH}) \mathrm{D}_{3}$ concentrations. The latter vitamin $\mathrm{D}$ metabolite is the widely accepted indicator of the vitamin $\mathrm{D}_{3}$ status of the human body (Hollis, 2005). The serum $25(\mathrm{OH}) \mathrm{D}_{3}$ concentrations change only in the order of weeks and months, such as the result of seasonal variations in sun exposure (Virtanen et al., 2011). This indicates that stimulation experiments with $1,25(\mathrm{OH})_{2} \mathrm{D}_{3}$ over a few hours, as performed in in vitro experiments, do not represent 


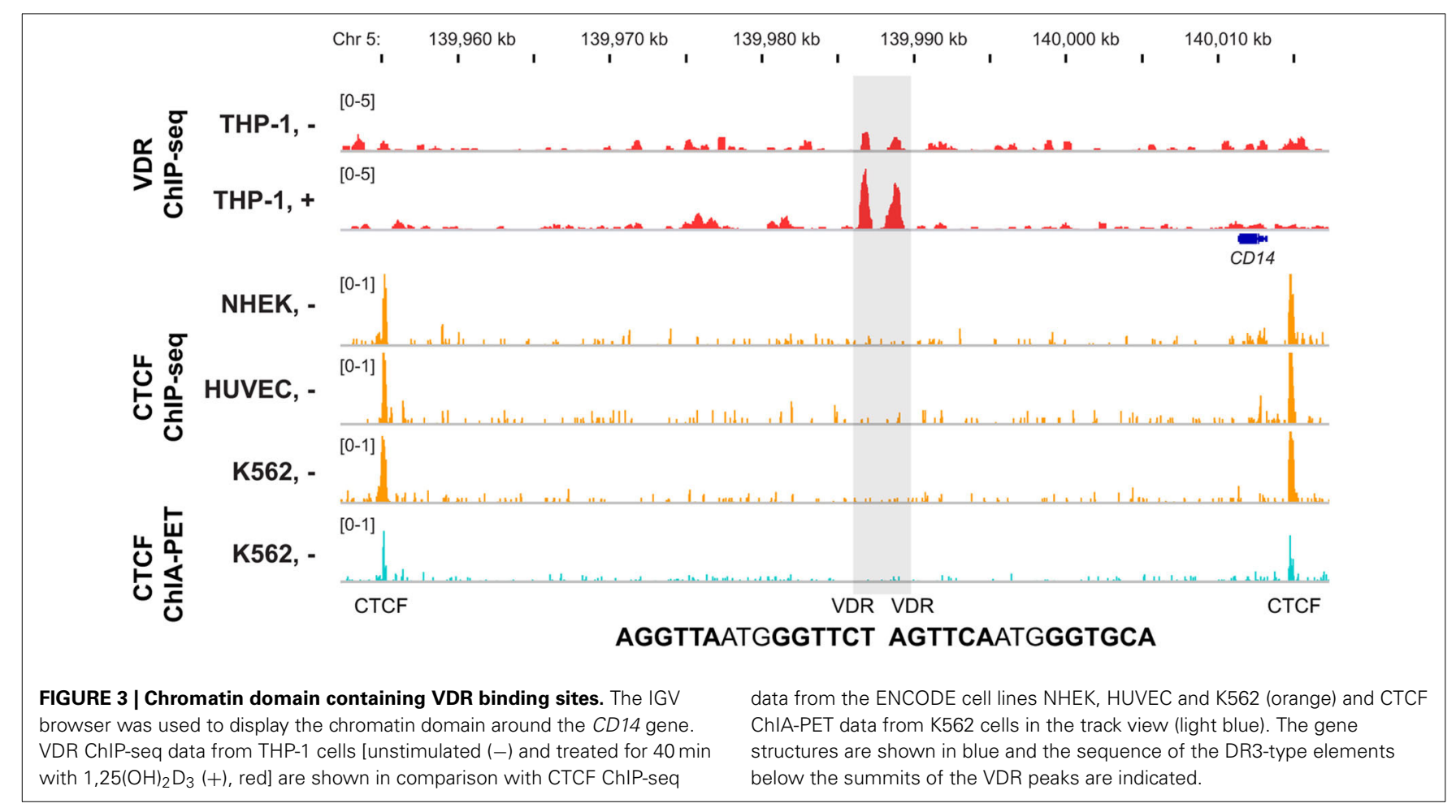

the physiological reality. In contrast, the effects of more longlasting changes of serum $25(\mathrm{OH}) \mathrm{D}_{3}$ concentrations should be considered. On a genome-wide level, this was investigated first with primary $\mathrm{T}$ cells isolated from nine human individuals with variant serum $25(\mathrm{OH}) \mathrm{D}_{3}$ concentrations (Handel et al., 2013). The number of the observed VDR ChIP-seq peaks, which varied between 200 and more than 7000 , correlated with the $25(\mathrm{OH}) \mathrm{D}_{3}$ levels of the individuals, i.e., the higher the circulating $25(\mathrm{OH}) \mathrm{D}_{3}$ concentrations, the more VDR loci were identified in $\mathrm{T}$ cells. Unfortunately, the raw data of this study is not available, i.e., a harmonized re-analysis in comparison with other published VDR ChIP-seq data cannot be performed. However, from the 14,044 unique VDR peaks reported for the sum of the nine individuals, only $442(3.1 \%)$ associated with a DR3-type sequence (based on HOMER score 7 settings).

Serum $25(\mathrm{OH}) \mathrm{D}_{3}$ concentrations vary widely from person to person based on (i) varied diet and sun exposure, (ii) different age and/or level of adiposity and (iii) genetic and epigenetic variations (Engelman et al., 2008; Orton et al., 2008; Snellman et al., 2009). The Institute of Medicine recommends a serum $25(\mathrm{OH}) \mathrm{D}_{3}$ level of $50 \mathrm{nM}$ (Institute-of-Medicine, 2011), but it is under debate, whether this is sufficient for every individual (Holick, 2007). In fact, a substantial proportion of the world's population could be considered as vitamin $\mathrm{D}$ deficient. This condition may accelerate age-related bone loss and morbidity from falls and fractures. In addition, vitamin D insufficiency is associated with a number of diseases, such as cancer, autoimmune disorders and all components of the metabolic syndrome (more details in the article by Bendik et al. in this issue).

This important medical problem guided to the question, whether an insight into the genome- and transcriptome-wide actions of VDR and $1,25(\mathrm{OH})_{2} \mathrm{D}_{3}$ can help in a more accurate evaluation of the human individual's responsiveness to, and needs for, vitamin D. A first approach in this direction was done by studying peripheral blood mononuclear cells (PBMCs) and adipose tissue biopsies from 71 elderly, pre-diabetic individuals, which participated in a 5-month vitamin $\mathrm{D}_{3}$ intervention trial (VitDmet) during Finnish winter (Carlberg et al., 2013). The changes in the mRNA expression of the primary $1,25(\mathrm{OH})_{2} \mathrm{D}_{3}$ target genes $C D 14$ and thrombomodulin $(T H B D)$, which had been identified in a recent comparative study as most reliable biomarkers (Standahl Olsen et al., 2013), in both PBMCs and fat samples were correlated with the alterations in the serum $25(\mathrm{OH}) \mathrm{D}_{3}$ levels of the 71 individuals. Interestingly, only for a subset of individuals significant correlations between the up-regulation of both genes and the intervention-induced raise in serum $25(\mathrm{OH}) \mathrm{D}_{3}$ concentrations were obtained. This suggests that, on a molecular level, not all study participants benefited from the vitamin $\mathrm{D}_{3}$ supplementation, because (i) they had already reached their individual optimal vitamin D status before the start of the intervention, (ii) they carry a genetic polymorphism making them less responsive to vitamin $\mathrm{D}_{3}$ or (iii) other undefined reasons (Carlberg et al., 2013). Interestingly, the categorization of the human individuals by their vitamin $\mathrm{D}$ responsiveness unmasked a negative correlation between changes in serum concentrations of $25(\mathrm{OH}) \mathrm{D}_{3}$ and the inflammation marker interleukin 6, i.e., the more responsive the study participants were to vitamin $\mathrm{D}_{3}$ supplementation, the lower was their inflammatory status. At present, a number of other primary $1,25(\mathrm{OH})_{2} \mathrm{D}_{3}$ target genes, which were highlighted in the comparison of VDR ChIP-seq data, are evaluated for their potential to serve as even better biomarkers 
for the vitamin D status of human individuals than CD14 and $T H B D$.

In summary, vitamin $\mathrm{D}$ deficiency may negatively contribute to a number of diseases. Genome-wide insight led to the use of mRNA expression changes of the genes CD14 and THBD as biomarkers for a molecular evaluation of vitamin $\mathrm{D}_{3}$ supplementation studies. The results allow a classification of human individuals based on their responsiveness to vitamin $\mathrm{D}_{3}$.

\section{CONCLUSIONS}

Genome-wide data on (i) the location of transcription factor binding sites in living cells, (ii) histone modifications and (iii) accessible chromatin, such as provided by ChIP-seq, DNase-seq and FAIRE-seq studies, have significantly changed the view on, and the understanding of the regulation of the entirety of the genes of our genome. This applies also to the transcription factor VDR, for which at present ChIP-seq data from six human cell lines and the $\mathrm{T}$ cells of nine human individuals are available. The abovementioned modern genome-wide techniques allow a more unbiased identification of transcription factor binding sites compared to previous studies, which were mostly focused on regions a few kb upstream of a primary $1,25(\mathrm{OH})_{2} \mathrm{D}_{3}$ target gene. VDR binding loci have now been shown to be localized equally likely up- and downstream of TSS regions in distances of even more than $1 \mathrm{Mb}$.

Genome-wide studies have confirmed DR3-type sequences as the preferential binding sites for VDR (most likely as a heterodimer with RXR), but only one in seven of the close to 20,000 known VDR binding loci carry such a motif. This is unanimously observed in all investigated cellular models. Therefore, there must be other types of binding motifs and partnering proteins that attract VDR to its genomic targets. These presently poorly understood alternative binding modes may explain some of VDR's function in the (trans)repression of its target genes. Moreover, the VDR cistrome seems to be largely cell-specific with only some 50 loci overlapping in all investigated models. However, these conserved sites could be fundamental entry ports of VDR to the human genome, which may serve as the unified core of the various pleiotropic functions of $1,25(\mathrm{OH})_{2} \mathrm{D}_{3}$.

The 22,000 detected VDR binding loci within six cell lines as well as the 14,000 peaks found in primary $\mathrm{T}$ cells from nine human individuals may be far more than what is needed to control the physiological actions of $1,25(\mathrm{OH})_{2} \mathrm{D}_{3}$, i.e., many sites may represent rather "noise" than having a specific function. Therefore, different approaches to categorize VDR loci are useful. It turned out that VDR binding sites that (i) carry a DR3-type sequence, (ii) show ligand-stimulated VDR association, (iii) co-locate with ligand-induced chromatin opening and (iv) are conserved between several cellular systems may play a more important role in mediating the functions of $1,25(\mathrm{OH})_{2} \mathrm{D}_{3}$ than the vast majority of other VDR sites that lack most of these properties. Therefore, for a cellular system of interest, the combination of (i) a genome-wide assessment of open chromatin by DNase-seq or FAIRE-seq, (ii) the monitoring of genomic VDR loci by ChIPseq and (iii) a screening for DR3-type sequences below the peak summits is an efficient tool for the prediction and identification of primary $1,25(\mathrm{OH})_{2} \mathrm{D}_{3}$ target genes.

\section{FUTURE PERSPECTIVES}

Although historically $1,25(\mathrm{OH})_{2} \mathrm{D}_{3}$ was understood to be a hormone controlling calcium homeostasis and bone formation, to date the genome-wide the actions of VDR are best understood and monitored in cells of the hematopoietic system. This emphasizes the impact of $1,25(\mathrm{OH})_{2} \mathrm{D}_{3}$ on the function of innate and adaptive immunity. There are first indications that the core actions of $1,25(\mathrm{OH})_{2} \mathrm{D}_{3}$ and its receptor VDR can be extrapolated from hematopoietic cells to other tissues and cell types of the human body (Carlberg et al., 2013). If this holds true, the vitamin $\mathrm{D}$ status and responsiveness of a human individual can be derived from the response of, for example, PBMCs. Technically, various types of leukocytes can be collected far easier from blood samples than any other tissue biopsy. Like a glucose tolerance test is used to monitor the functionality of the carbohydrate metabolism of an individual, there may be in the future a higher dose vitamin $\mathrm{D}_{3}$ challenge test, where the (epi)genomic and transcriptomic profiles of leukocytes before and after supplementation are measured. Routine measurements of healthy individuals may also in the future more likely be based on a few selected biomarkers, such as CD14 and THBD, while more complex scenarios in disease settings, such as cancer or autoimmune diseases, will be assessed genome- or transcriptome-wide. In the same way, basic research on $1,25(\mathrm{OH})_{2} \mathrm{D}_{3}$ and VDR will shift more and more from cell culture models to primary tissues and cell types and will eventually reach the single cell level.

\section{ACKNOWLEDGMENTS}

The author thanks Dr. Sabine Seuter for critical reading of the manuscript and the Academy of Finland and the Juselius Foundation for support.

\section{REFERENCES}

Bouillon, R., and Suda, T. (2014). Vitamin D: calcium and bone homeostasis during evolution. Bonekey Rep. 3:480. doi: 10.1038/bonekey.2013.214

Carlberg, C., Bendik, I., Wyss, A., Meier, E., Sturzenbecker, L. J., Grippo, J. F., and Hunziker, W. (1993). Two nuclear signalling pathways for vitamin D. Nature 361, 657-660.

Carlberg, C., and Campbell, M. J. (2013). Vitamin D receptor signaling mechanisms: integrated actions of a well-defined transcription factor. Steroids 78, 127-136. doi: 10.1016/j.steroids.2012.10.019

Carlberg, C., and Molnár, F. (2012). Current status of vitamin D signaling and its therapeutic applications. Curr. Top. Med. Chem. 12, 528-547. doi: $10.2174 / 156802612799436623$

Carlberg, C., Seuter, S., De Mello, V. D., Schwab, U., Voutilainen, S., Pulkki, K., et al. (2013). Primary vitamin D target genes allow a categorization of possible benefits of vitamin $\mathrm{D}_{3}$ supplementation. PLoS ONE 8:e71042. doi: 10.1371/journal.pone.0071042

Crawford, G. E., Holt, I. E., Whittle, J., Webb, B. D., Tai, D., Davis, S., et al. (2006). Genome-wide mapping of DNase hypersensitive sites using massively parallel signature sequencing (MPSS). Genome Res. 16, 123-131. doi: 10.1101/gr.4074106

Deluca, H. F. (2004). Overview of general physiologic features and functions of vitamin D. Am. J. Clin. Nutr. 80, 1689S-1696S.

Ding, N., Yu, R. T., Subramaniam, N., Sherman, M. H., Wilson, C., Rao, R., et al. (2013). A vitamin D receptor/SMAD genomic circuit gates hepatic fibrotic response. Cell 153, 601-613. doi: 10.1016/j.cell.2013.03.028

Encode-Project-Consortium, Bernstein, B. E., Birney, E., Dunham, I., Green, E. D., Gunter, C., and Snyder, M. (2012). An integrated encyclopedia of DNA elements in the human genome. Nature 489, 57-74. doi: 10.1038/nature11247

Engelman, C. D., Fingerlin, T. E., Langefeld, C. D., Hicks, P. J., Rich, S. S., Wagenknecht, L. E., et al. (2008). Genetic and environmental determinants 
of 25-hydroxyvitamin D and 1,25-dihydroxyvitamin D levels in Hispanic and African Americans. J. Clin. Endocrinol. Metab. 93, 3381-3388. doi: 10.1210/jc.2007-2702

Fretz, J. A., Zella, L. A., Kim, S., Shevde, N. K., and Pike, J. W. (2007). 1,25Dihydroxyvitamin $\mathrm{D}_{3}$ induces expression of the Wnt signaling co-regulator LRP5 via regulatory elements located significantly downstream of the gene's transcriptional start site. J. Steroid Biochem. Mol. Biol. 103, 440-445. doi: 10.1016/j.jsbmb.2006.11.018

Fullwood, M. J., Liu, M. H., Pan, Y. F., Liu, J., Xu, H., Mohamed, Y. B., et al. (2009). An oestrogen-receptor-alpha-bound human chromatin interactome. Nature 462, 58-64. doi: 10.1038/nature08497

Furey, T. S. (2012). ChIP-seq and beyond: new and improved methodologies to detect and characterize protein-DNA interactions. Nat. Rev. Genet. 13, 840-852. doi: $10.1038 / \mathrm{nrg} 3306$

Giresi, P. G., Kim, J., McDaniell, R. M., Iyer, V. R., and Lieb, J. D. (2007). FAIRE (Formaldehyde-Assisted Isolation of Regulatory Elements) isolates active regulatory elements from human chromatin. Genome Res. 17, 877-885. doi: $10.1101 /$ gr.5533506

Handel, A. E., Sandve, G. K., Disanto, G., Berlanga-Taylor, A. J., Gallone, G., Hanwell, H., et al. (2013). Vitamin D receptor ChIP-seq in primary CD4 ${ }^{+}$cells: relationship to serum 25-hydroxyvitamin D levels and autoimmune disease. BMC Med 11:163. doi: 10.1186/1741-7015-11-163

Haussler, M. R., Haussler, C. A., Jurutka, P. W., Thompson, P. D., Hsieh, J. C., Remus, L. S., et al. (1997). The vitamin D hormone and its nuclear receptor: molecular actions and disease states. J. Endocrinol. 154(Suppl.), S57-S73.

Haussler, M. R., Whitfield, G. K., Kaneko, I., Haussler, C. A., Hsieh, D., Hsieh, J.-C., and Jurutka, P. W. (2013). Molecular mechanisms of vitamin D action. Calcif. Tissue Int. 92, 77-98. doi: 10.1007/s00223-012-9619-0

Heikkinen, S., Väisänen, S., Pehkonen, P., Seuter, S., Benes, V., and Carlberg, C. (2011). Nuclear hormone $1 \alpha, 25$-dihydroxyvitamin $D_{3}$ elicits a genome-wide shift in the locations of VDR chromatin occupancy. Nucleic Acids Res. 39, 9181-9193. doi: 10.1093/nar/gkr654

Heinz, S., Benner, C., Spann, N., Bertolino, E., Lin, Y. C., Laslo, P., et al. (2010). Simple combinations of lineage-determining transcription factors prime cisregulatory elements required for macrophage and B cell identities. Mol. Cell 38, 576-589. doi: 10.1016/j.molcel.2010.05.004

Hochberg, Z., and Templeton, A. R. (2010). Evolutionary perspective in skin color, vitamin D and its receptor. Hormones (Athens) 9, 307-311. doi: 10.14310/horm.2002.1281

Holick, M. F. (2004). Sunlight and vitamin D for bone health and prevention of autoimmune diseases, cancers, and cardiovascular disease. Am. J. Clin. Nutr. 80, 1678S-1688S.

Holick, M. F. (2007). Vitamin D deficiency. N. Engl. J. Med. 357, 266-281. doi: 10.1056/NEJMra070553

Holick, M. F. (2011). Vitamin D: evolutionary, physiological and health perspectives. Curr. Drug Targets 12, 4-18. doi: 10.2174/138945011793591635

Hollis, B. W. (2005). Circulating 25-hydroxyvitamin D levels indicative of vitamin D sufficiency: implications for establishing a new effective dietary intake recommendation for vitamin D. J. Nutr. 135, 317-322.

Institute-of-Medicine. (2011). Dietary Reference Intakes for Calcium and Vitamin D. Washington, DC: National Academies Press.

Jones, G., Strugnell, S. A., and Deluca, H. F. (1998). Current understanding of the molecular actions of vitamin D. Physiol. Rev. 78, 1193-1231.

Kadauke, S., and Blobel, G. A. (2009). Chromatin loops in gene regulation. Biochim. Biophys. Acta 1789, 17-25. doi: 10.1016/j.bbagrm.2008.07.002

Kim, S., Yamazaki, M., Zella, L. A., Shevde, N. K., and Pike, J. W. (2006) Activation of receptor activator of NF-kappaB ligand gene expression by 1,25 dihydroxyvitamin $\mathrm{D}_{3}$ is mediated through multiple long-range enhancers. Mol. Cell Biol. 26, 6469-6486. doi: 10.1128/MCB.00353-06

Kriebitzsch, C., Verlinden, L., Eelen, G., Van Schoor, N. M., Swart, K., Lips, P., et al. (2011). 1,25-dihydroxyvitamin $\mathrm{D}_{3}$ influences cellular homocysteine levels in murine preosteoblastic MC3T3-E1 cells by direct regulation of cystathionine beta-synthase. J. Bone Miner. Res. 26, 2991-3000. doi: 10.1002/jbmr.493

Mangelsdorf, D. J., Thummel, C., Beato, M., Herrlich, P., Schütz, G., Umesono, K., et al. (1995). The nuclear receptor superfamily: the second decade. Cell 83, 835-839.

Maston, G. A., Landt, S. G., Snyder, M., and Green, M. R. (2012). Characterization of enhancer function from genome-wide analyses. Annu. Rev. Genomics Hum. Genet. 13, 29-57. doi: 10.1146/annurev-genom-090711-163723
Meyer, M. B., Goetsch, P. D., and Pike, J. W. (2010). A downstream intergenic cluster of regulatory enhancers contributes to the induction of CYP24A1 expression by $1 \alpha, 25$-dihydroxyvitamin $\mathrm{D}_{3}$. J. Biol. Chem. 285, 15599-15610. doi: 10.1074/jbc.M110.119958

Meyer, M. B., Goetsch, P. D., and Pike, J. W. (2012). VDR/RXR and TCF4/betacatenin cistromes in colonic cells of colorectal tumor origin: impact on c-FOS and c-MYC gene expression. Mol. Endocrinol. 26, 37-51. doi: 10.1210/me.20111109

Meyer, M. B., Watanuki, M., Kim, S., Shevde, N. K., and Pike, J. W. (2006). The human transient receptor potential vanilloid type 6 distal promoter contains multiple vitamin D receptor binding sites that mediate activation by 1,25 dihydroxyvitamin $\mathrm{D}_{3}$ in intestinal cells. Mol. Endocrinol. 20, 1447-1461. doi: 10.1210/me.2006-0031

Misteli, T. (2007). Beyond the sequence: cellular organization of genome function. Cell 128, 787-800. doi: 10.1016/j.cell.2007.01.028

Mohn, F., and Schubeler, D. (2009). Genetics and epigenetics: stability and plasticity during cellular differentiation. Trends Genet. 25, 129-136. doi: 10.1016/j.tig.2008.12.005

Molnár, F., Peräkylä, M., and Carlberg, C. (2006). Vitamin D receptor agonists specifically modulate the volume of the ligand-binding pocket. J. Biol. Chem. 281, 10516-10526. doi: 10.1074/jbc.M513609200

Moras, D., and Gronemeyer, H. (1998). The nuclear receptor ligand-binding domain: structure and function. Curr. Opin. Cell Biol. 10, 384-391.

Narlikar, G. J., Fan, H. Y., and Kingston, R. E. (2002). Cooperation between complexes that regulate chromatin structure and transcription. Cell 108, 475-487. doi: 10.1016/S0092-8674(02)00654-2

Norman, A. W. (2008). From vitamin D to hormone D: fundamentals of the vitamin D endocrine system essential for good health. Am. J. Clin. Nutr. 88, 491S-499S.

Orlando, V. (2000). Mapping chromosomal proteins in vivo by formaldehydecrosslinked-chromatin immunoprecipitation. Trends Biochem. Sci. 25, 99-104. doi: 10.1016/S0968-0004(99)01535-2

Orton, S. M., Morris, A. P., Herrera, B. M., Ramagopalan, S. V., Lincoln, M. R., Chao, M. J., et al. (2008). Evidence for genetic regulation of vitamin D status in twins with multiple sclerosis. Am. J. Clin. Nutr. 88, 441-447.

Park, P. J. (2009). ChIP-seq: advantages and challenges of a maturing technology. Nat. Rev. Genet. 10, 669-680. doi: 10.1038/nrg2641

Perissi, V., Jepsen, K., Glass, C. K., and Rosenfeld, M. G. (2010). Deconstructing repression: evolving models of co-repressor action. Nat. Rev. Genet. 11, 109-123. doi: $10.1038 / \operatorname{nrg} 2736$

Polly, P., Herdick, M., Moehren, U., Baniahmad, A., Heinzel, T., and Carlberg, C. (2000). VDR-Alien: a novel, DNA-selective vitamin $\mathrm{D}_{3}$ receptor-corepressor partnership. FASEB J. 14, 1455-1463. doi: 10.1096/fj.14.10.1455

Ramagopalan, S. V., Heger, A., Berlanga, A. J., Maugeri, N. J., Lincoln, M. R., Burrell, A., et al. (2010). A ChIP-seq defined genome-wide map of vitamin D receptor binding: associations with disease and evolution. Genome Res. 20, 1352-1360. doi: 10.1101/gr.107920.110

Razin, A. (1998). CpG methylation, chromatin structure and gene silencing-a three-way connection. EMBO J. 17, 4905-4908.

Robinson, J. T., Thorvaldsdottir, H., Winckler, W., Guttman, M., Lander, E. S., Getz, G., and Mesirov, J. P. (2011). Integrative genomics viewer. Nat. Biotechnol. 29, 24-26. doi: 10.1038/nbt.1754

Ryynänen, J., Seuter, S., Campbell, M. J., and Carlberg, C. (2013). Gene regulatory scenarios of primary 1,25-dihydroxyvitamin $\mathrm{D}_{3}$ target genes in a human myeloid leukemia cell line. Cancers 5, 1221-1241. doi: 10.3390/cancers50 41221

Saramäki, A., Banwell, C. M., Campbell, M. J., and Carlberg, C. (2006). Regulation of the human $\mathrm{p} 21^{(\text {waf } 1 / \text { cip } 1)}$ gene promoter via multiple binding sites for $\mathrm{p} 53$ and the vitamin $\mathrm{D}_{3}$ receptor. Nucleic Acids Res. 34, 543-554. doi: 10.1093/nar/ gkj460

Saramäki, A., Diermeier, S., Kellner, R., Laitinen, H., Väisänen, S., and Carlberg, C. (2009). Cyclical chromatin looping and transcription factor association on the regulatory regions of the p21 (CDKN1A) gene in response to $1 \alpha, 25$-dihydroxyvitamin $\mathrm{D}_{3}$. J. Biol. Chem. 284, 8073-8082. doi: 10.1074/jbc.M808090200

Schmidt, D., Schwalie, P. C., Wilson, M. D., Ballester, B., Gonçalves, A., Kutter, C., et al. (2012). Waves of retrotransposon expansion remodel genome organization and CTCF binding in multiple mammalian lineages. Cell 148, 335-348. doi: 10.1016/j.cell.2011.11.058 
Schüle, R., Umesono, K., Mangelsdorf, D. J., Bolado, J., Pike, J. W., and Evans, R. M. (1990). Jun-Fos and receptors for vitamins A and D recognize a common response element in the human osteocalcin gene. Cell 61, 497-504.

Seuter, S., Neme, A., and Carlberg, C. (2014). Characterization of genomic vitamin D receptor binding sites through chromatin looping and opening. PLoS ONE 9. doi: 10.1371/journal.pone.0096184

Seuter, S., Pehkonen, P., Heikkinen, S., and Carlberg, C. (2013). Dynamics of $1 \alpha, 25$-dihydroxyvitamin D-dependent chromatin accessibility of early vitamin D receptor target genes. Biochim. Biophys. Acta 1829, 1266-1275. doi: 10.1016/j.bbagrm.2013.10.003

Seuter, S., Väisänen, S., Radmark, O., Carlberg, C., and Steinhilber, D. (2007). Functional characterization of vitamin D responding regions in the human 5lipoxygenase gene. Biochim. Biophys. Acta 1771, 864-872. doi: 10.1016/j.bbalip. 2007.04.007

Shaffer, P. L., and Gewirth, D. T. (2002). Structural basis of VDR-DNA interactions on direct repeat response elements. EMBO J. 21, 2242-2252. doi: 10.1093/emboj/21.9.2242

Shaffer, P. L., and Gewirth, D. T. (2004). Structural analysis of RXR-VDR interactions on DR3 DNA. J. Steroid Biochem. Mol. Biol. 89-90, 215-219. doi: 10.1016/j.jsbmb.2004.03.084

Sierra, J., Villagra, A., Paredes, R., Cruzat, F., Gutierrez, S., Javed, A., et al. (2003). Regulation of the bone-specific osteocalcin gene by p300 requires Runx2/Cbfal and the vitamin $\mathrm{D}_{3}$ receptor but not $\mathrm{p} 300$ intrinsic histone acetyltransferase activity. Mol. Cell Biol. 23, 3339-3351. doi: 10.1128/MCB.23.9.33393351.2003

Sinkkonen, L., Malinen, M., Saavalainen, K., Väisänen, S., and Carlberg, C. (2005). Regulation of the human cyclin $C$ gene via multiple vitamin $D_{3}$ responsive regions in its promoter. Nucleic Acids Res. 33, 2440-2451. doi: 10.1093/nar/gki502

Snellman, G., Melhus, H., Gedeborg, R., Olofsson, S., Wolk, A., Pedersen, N. L., et al. (2009). Seasonal genetic influence on serum 25-hydroxyvitamin D levels: a twin study. PLoS ONE 4:e7747. doi: 10.1371/journal.pone.0007747

Sone, T., Ozono, K., and Pike, J. W. (1991). A 55-kilodalton accessory factor facilitates vitamin D receptor DNA binding. Mol Endocrinol 5, 1578-1586.

Standahl Olsen, K., Rylander, C., Brustad, M., Aksnes, L., and Lund, E. (2013). Plasma 25 hydroxyvitamin D level and blood gene expression profiles: a crosssectional study of the Norwegian women and cancer post-genome cohort. Eur. J. Clin. Nutr. 67, 773-778. doi: 10.1038/ejcn.2013.53

Talbert, P. B., and Henikoff, S. (2006). Spreading of silent chromatin: inaction at a distance. Nat. Rev. Genet. 7, 793-803. doi: 10.1038/nrg1920

Tolon, R. M., Castillo, A. I., Jimenez-Lara, A. M., and Aranda, A. (2000). Association with ets-1 causes ligand- and AF2-independent activation of nuclear receptors. Mol. Cell Biol. 20, 8793-8802. doi: 10.1128/MCB.20.23.87938802.2000

Toropainen, S., Väisänen, S., Heikkinen, S., and Carlberg, C. (2010). The down-regulation of the human MYC gene by the nuclear hormone $1 \alpha, 25$ dihydroxyvitamin $\mathrm{D}_{3}$ is associated with cycling of corepressors and histone deacetylases. J. Mol. Biol. 400, 284-294. doi: 10.1016/j.jmb.2010.05.031

Tuoresmäki, P., Väisänen, S., Neme, A., Heikkinen, S., and Carlberg, C. (2014). Patterns of genome-wide VDR locations. PLOS ONE 9. doi: 10.1371/journal. pone.0096105
Turunen, M. M., Dunlop, T. W., Carlberg, C., and Väisänen, S. (2007). Selective use of multiple vitamin $\mathrm{D}$ response elements underlies the $1 \alpha, 25$-dihydroxyvitamin $\mathrm{D}_{3}$-mediated negative regulation of the human CYP27B1 gene. Nucleic Acids Res 35, 2734-2747. doi: 10.1093/nar/gkm179

Umesono, K., Murakami, K. K., Thompson, C. C., and Evans, R. M. (1991). Direct repeats as selective response elements for the thyroid hormone, retinoic acid, and vitamin $\mathrm{D}_{3}$ receptors. Cell 65, 1255-1266.

Väisänen, S., Dunlop, T. W., Sinkkonen, L., Frank, C., and Carlberg, C. (2005). Spatio-temporal activation of chromatin on the human CYP24 gene promoter in the presence of 1 $\alpha, 25$-dihydroxyvitamin $\mathrm{D}_{3}$. J. Mol. Biol. 350, 65-77. doi: 10.1016/j.jmb.2005.04.057

Van Bortle, K., and Corces, V. G. (2013). The role of chromatin insulators in nuclear architecture and genome function. Curr. Opin. Genet. Dev. 23, 212-218. doi: 10.1016/j.gde.2012.11.003

Vaquerizas, J. M., Kummerfeld, S. K., Teichmann, S. A., and Luscombe, N. M. (2009). A census of human transcription factors: function, expression and evolution. Nat. Rev. Genet. 10, 252-263. doi: 10.1038/ $\operatorname{nrg} 2538$

Virtanen, J. K., Nurmi, T., Voutilainen, S., Mursu, J., and Tuomainen, T. P. (2011). Association of serum 25-hydroxyvitamin $\mathrm{D}$ with the risk of death in a general older population in Finland. Eur. J. Nutr. 50, 305-312. doi: 10.1007/s00394-0100138-3

Wang, Y., Zhu, J., and Deluca, H. F. (2012). Where is the vitamin D receptor? Arch. Biochem. Biophys. 523, 123-133. doi: 10.1016/j.abb.2012.04.001

Zaret, K. S., and Carroll, J. S. (2011). Pioneer transcription factors: establishing competence for gene expression. Gen. Dev. 25, 2227-2241. doi: 10.1101/gad.176826.111

Zella, L. A., Kim, S., Shevde, N. K., and Pike, J. W. (2006). Enhancers located within two introns of the vitamin $\mathrm{D}$ receptor gene mediate transcriptional autoregulation by 1,25-dihydroxyvitamin $\mathrm{D}_{3}$. Mol. Endocrinol. 20, 1231-1247. doi: 10.1210/me.2006-0015

Zella, L. A., Meyer, M. B., Nerenz, R. D., Lee, S. M., Martowicz, M. L., and Pike, J. W. (2010). Multifunctional enhancers regulate mouse and human vitamin D receptor gene transcription. Mol. Endocrinol. 24, 128-147. doi: $10.1210 /$ me.2009-0140

Conflict of Interest Statement: The authors declare that the research was conducted in the absence of any commercial or financial relationships that could be construed as a potential conflict of interest.

Received: 14 February 2014; accepted: 10 April 2014; published online: 29 April 2014. Citation: Carlberg C (2014) Genome-wide (over)view on the actions of vitamin D. Front. Physiol. 5:167. doi: 10.3389/fphys.2014.00167

This article was submitted to Integrative Physiology, a section of the journal Frontiers in Physiology.

Copyright (C) 2014 Carlberg. This is an open-access article distributed under the terms of the Creative Commons Attribution License (CC BY). The use, distribution or reproduction in other forums is permitted, provided the original author(s) or licensor are credited and that the original publication in this journal is cited, in accordance with accepted academic practice. No use, distribution or reproduction is permitted which does not comply with these terms. 